Chirurg 2017 $\cdot 88: 467-468$

DOI 10.1007/s00104-017-0423-2

(c) Springer Medizin Verlag GmbH 2017

CrossMark

\section{U. Settmacher}

Klinik für Allgemein-, Viszeral- und Gefäßchirurgie, Universitätsklinikum Jena, Jena, Deutschland

\title{
Neues aus der minimalinvasiven hepatopankreatobiliären Chirurgie
}

Ziele der chirurgischen Behandlung von Erkrankungen der Leber, Gallenwege und der Bauchspeicheldrüse sind, ein hohes Langzeitüberleben, Lebensqualität und beim Malignom Tumorfreiheit für die Patienten zu ermöglichen. Diese Operationen minimalinvasiv durchzuführen, versucht man schon längere Zeit. In Deutschland besteht im Vergleich zu anderen Ländern aber eine gewisse $\mathrm{Zu}$ rückhaltung in der Verbreitung dieser Zugangstechnik und der Ausdehnung auf komplexe Operationen. Gründe dafür sind sicher zunächst die Frage der Bergung großer Tumorpräparate, die dann doch einen größeren Bergungsschnitt erfordern, lange Operationszeiten bei komplexen Eingriffen, die gerade in der Trainings- bzw. Lernphase des Operateurs bestehen, die unzureichende bzw. komplett fehlende Haptik, die durch andere sensorielle Informationen kompensiert werden muss, und die teilweise nicht ausreichend ausgereiften Instrumente, die Rekonstruktionen oder Parenchymdissektionen erschweren. Schließlich ist ein Vorteil der minimalinvasiven gegenüber der konventionellen Chirurgie in diesem Bereich bis auf die Cholezystektomie noch nicht erwiesen. Ein weiterer Punkt sind die höheren Materialkosten der laparoskopischen und erst recht der roboterassistierten Verfahren.

Trotzdem entwickeln sich durch den Enthusiasmus begeisterter und visionärer Chirurgen und auch durch Promotion der Industrie diese Techniken in der Leber- und Pankreaschirurgie. Es werden zurzeit unter Bedacht aller Pros und Cons einerseits ein Indikationsspektrum für laparoskopische und roboterassistierte Operationen, die sich auch m. E. in ihren technischen Möglichkeiten erheblich unterscheiden, konturiert und andererseits an der technischen Verbesserung und dem Training der Operateure intensiv gearbeitet.

Nachdem wir in einem Vorheft von Der Chirurg versucht haben, von internationalen Experten zu Entwicklungen der roboterassistierten Viszeralchirurgie $\mathrm{zu}$ informieren, soll in diesem Heft von deutschen Vertretern der minimalinvasiven hepatopankreatischen Chirurgie ein aktuelles Bild dieser Verfahren sowie Chancen der Entwicklung und mögliche Probleme und deren Entwicklung auch in Deutschland aufgezeichnet werden.

\section{》) In Japan sind laparoskopische Minor-Leberresektionen schon fast Standard}

M. Schön und C. Justinger berichten zum Stand der laparoskopischen Leberchirurgie und illustrieren die Fortschritte anhand eines eigenen Videos zum laparoskopischen ALPPS („associating liver partition and portal vein ligation"). $\mathrm{Ne}$ ben den internationalen Erfahrungen werden der Stand der Evidienz und technische Details vorgestellt. Nach zwei internationalen Konsensuskonferenzen werden inzwischen in Japan laparoskopische Minor-Resektionen schon fast als Standard betrachtet. Zur Feststellung der Evidenz laufen derzeit zwei internationale Studien. Größe und Lage von Raumforderungen sind heute die entscheidenden Kriterien, ob eine laparoskopische Resektion durchgeführt werden kann. Die Möglichkeit wiederholter Resektionen bei nur geringer 


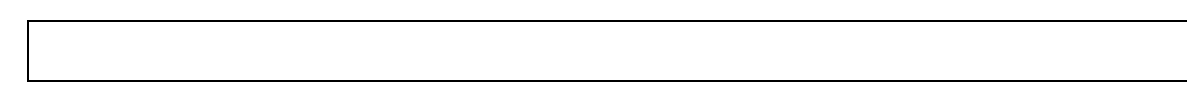

Verwachsungsbildung erhöht die Attraktivität der Methode.

H. Aselmann et al. berichten zur roboterassistierten Leberchirurgie. Sie zeigen, dass man roboterassistiert einige der Limitierungen der laparoskopischen Leberchirurgie überwinden kann. Schwierig ist bisher die Technik der Parenchymdissektion. So erfolgte dies in verschiedenen Arbeiten laparoskopisch oft mit der Ultraschallschere, während roboterassistiert häufiger die Clamp-crushTechnik verwendet wurde. Neben den Erfahrungen aus Kiel und Erlangen wird eine Umfrage zur roboterassistierten Leberchirurgie in Deutschland gezeigt und Perspektiven der Roboterchirurgie für ALPPS, Lebendspende sowie lokalablative Verfahren bzw. die lokoregionale Chemotherapie vorgestellt.

\section{》) Laparoskopische Eingriffe am Pankreaskopf haben eine ausgeprägte Lernkurve}

D. Bausch und T. Keck informieren zur laparoskopischen Pankreaschirurgie. Neben vielen Vorteilen sind aber prospektive Studien, welche eine onkologische Gleichwertigkeit $\mathrm{zu}$ offenen Verfahren zeigen noch durchzuführen. Erste Studiendaten zur Pankreaskopfresektion hierzu zeigen eine Gleichwertigkeit. Im Gegensatz zu vielen anderen laparoskopischen Eingriffen weist die Pankreaskopfresektion aber eine sehr ausgeprägte Lernkurve auf. Die laparoskopische Pankreaslinksresektion bei benignen Veränderungen darf inzwischen fast als Standardeingriff betrachtet werden. Sie ist der technisch am einfachsten durchführbare, minimalinvasive Eingriff am Pankreas.

B. Müssle et al. berichten zur roboterassistierten Pankreaschirurgie. Dabei scheint der Roboter gerade bei Pankreaskopfresektionen Vorteile gegenüber der laparoskopischen Technik zu haben. Hier besticht, wie sonst auch in der roboterassistierten Chirurgie, die bessere Möglichkeit und schnellere Erlernbarkeit von Organ- oder Gefäßanastomosen. Metaanalysen zur roboterassistierten Pankreasresektion attestieren die Sicherheit und Machbarkeit der Technik bei selektio- nierten Patienten. Die Autoren beschreiben die eigene Technik und geben Hinweise zur Lernkurve.

Insgesamt meine ich, geben diese Beiträge eine gelungene Übersicht zum aktuellen Stand der minimalinvasiven Chirurgie der Leber und der Bauchspeicheldrüse. Ich wünsche Ihnen Spaß beim Lesen und Anregung für das eigene Tun.

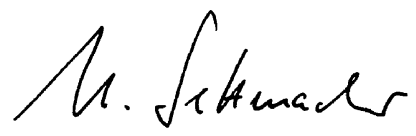

Prof. Dr. Utz Settmacher

\section{Korrespondenzadresse}

\section{Prof. Dr. U. Settmacher}

Klinik für Allgemein-, Viszeral- und Gefäßchirurgie, Universitätsklinikum Jena Erlanger Allee 101, 07740 Jena, Deutschland utz.settmacher@med.uni-jena.de

Interessenkonflikt. U. Settmacher gibt an, dass kein Interessenkonflikt besteht.
In eigener Sache

Autoren

WERKSTATT

GRATIS

Ein Service für Autoren von Springer Medizin

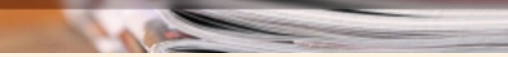

\section{Fortbildungen für Autoren und Gutachter}

Die ersten Veröffentlichungen sind für jeden Mediziner ein wichtiger Schritt in seiner Karriere als Wissenschaftler. Wissenschaftliche Artikel sind entscheidend dafür, dass die eigene Arbeit in der Community wahrgenommen wird. Es geht darum, die eigenen Ideen national und international auszutauschen und sicherzustellen, dass die Ergebnisse Wirkung erzielen. Die Online-Kurse der Autorenwerkstatt helfen, sich leicht einen Überblick über das Schreiben, Einreichen, Begutachten und Veröffentlichen eines Manuskripts zu verschaffen.

5 Online-Kurse zu den wichtigsten Standards des wissenschaftlichen Publizierens:

- Wie verfasse ich ein Manuskript?

- Writing in English für deutschsprachige Autoren

- Wie funktionieren Publikation und Begutachtung?

- Anleitung zur Open-AccessVeröffentlichung

- Leitfaden zur Peer-ReviewBegutachtung

Für alle, die auf SpringerMedizin.de registriert sind!

Jetzt gratis fortbilden unter www.springermedizin.de/ autorenwerkstatt/ 